\title{
Down-regulation of the transcription factor snail in the placentas of patients with preeclampsia and in a rat model of preeclampsia
}

Larisa Fedorova $^{1 *}$, Cara Gatto-Weis ${ }^{2}$, Sleiman Smaili ${ }^{3}$, Nauman Khurshid ${ }^{3}$, Joseph I Shapiro ${ }^{1}$, Deepak Malhotra ${ }^{1}$ and Terrence Horrigan ${ }^{3}$

\begin{abstract}
Background: Placental malfunction in preeclampsia is believed to be a consequence of aberrant differentiation of trophoblast lineages and changes in utero-placental oxygenation. The transcription factor Snail, a master regulator molecule of epithelial-mesenchymal transition in embryonic development and in cancer, is shown to be involved in trophoblast differentiation as well. Moreover, Snail can be controlled by oxidative stress and hypoxia. Therefore, we examined the expression of Snail and its downstream target, e-cadherin, in human normal term, preterm and preeclamptic placentas, and in pregnant rats that developed preeclampsia-like symptoms in the response to a 20fold increase in sodium intake.
\end{abstract}

Methods: Western blotting analysis was used for comparative expression of Snail and e- cadherin in total protein extracts. Placental cells expressing Snail and e-cadherin were identified by immunohistochemical double-labeling technique.

Results: The levels of Snail protein were decreased in human preeclamptic placentas by $30 \%(p<0.01)$ compared to normal term, and in the rat model by $40 \%(p<0.001)$ compared to control placentas. In preterm placentas, the levels of Snail expression varied, yet there was a strong trend toward statistical significance between preterm and preeclamptic placentas. In humans, e-cadherin protein level was 30\% higher in preeclamptic $(p<0.05)$ placentas and similarly, but not significantly $(p=0.1)$, high in the preterm placentas compared to normal term. In the rat model of preeclampsia, e-cadherin was increased by $60 \%(p<0.01)$. Immunohistochemical examination of human placentas demonstrated Snail-positive staining in the nuclei of the villous trophoblasts and mesenchymal cells and in the invasive trophoblasts of the decidua. In the rat placenta, the majority of Snail positive cells were spongiotrophoblasts of the junctional zone, while in the labyrinth, Snail-positive sinusoidal giant trophoblasts cells were found in some focal areas located close to the junctional zone.

Conclusion: We demonstrated that human preeclampsia and the salt-induced rat model of preeclampsia are associated with the reduced levels of Snail protein in placenta. Down-regulation of the transcription factor Snail in placental progenitor cell lineages, either by intrinsic defects and/or by extrinsic and maternal factors, may affect normal placenta development and function and thus contribute to the pathology of preeclampsia.

Keywords: Preeclampsia, Placenta, Snail, Trophoblast, E-cadherin

\footnotetext{
* Correspondence: larisa.fedorova@utoledo.edu

${ }^{1}$ Department of Medicine, University of Toledo School of Medicine, Toledo,

$\mathrm{OH}$ 43614, USA

Full list of author information is available at the end of the article
} 


\section{Background}

Preeclampsia, a devastating, life-threatening, human pregnancy complication, develops in $3-10 \%$ of pregnancies. Clinical symptoms include a sudden onset of hypertension accompanied by proteinuria, edema and often fetal growth restriction [1]. The placenta of patients with preeclampsia is malformed [2]. It has been hypothesized that abnormalities in trophoblast function may contribute to placental defects associated with preeclampsia [3]. An increased proliferation of progenitor villous cytotrophoblastic cells (VCT) and an augmented apoptosis of the terminally differentiated syncytiotrophoblast (SynTB) results in thinning and distortion of the syncytial layer, and in the appearance of multinucleated syncytial buds $[2,4,5]$. The villous injury in preeclampsia is associated with poor perfusion/oxygenation of the intervillous space, utero-placental hypoxia, and oxidative stress [6,7]. Proper oxygenation of the placenta is achieved through remodeling of the maternal spiral arteries by a population of VCTs which, upon contact with the decidua, acquire an invasive phenotype and are hence called extravillous trophoblast (EVT). In preeclampsia, EVT invasion is limited and vascular transformation is incomplete [2]. Transformation of polarized, non-motile, proliferative epithelia-like VCT into highly invasive EVT requires loss of polarity, down-regulation of epithelia-specific adhesion molecules, up-regulation of extracellular matrix receptors, and de novo expression of matrix degradation proteins [8]. This transformation to some extent resembles the process of epithelialmesenchymal transition (EMT), which is common in embryonic development and in metastastic cancers [9]. EMT is commonly initiated by down-regulation of the adhesive junction protein, e-cadherin. Similarly, in normal placenta, invasive and migratory, individual and aggregated EVTs exhibit reduced, discontinuous expression of e-cadherin [10]. Conversely, in preeclampsia, EVTs fail to down-regulate e-cadherin during the initiation of invasion in anchoring cell columns, and also during the fusion of the giant multinucleated cells in the inner myometrium [11-13].

In embryogenesis and cancer, e-cadherin expression is under the control of the Wnt pathways and/or the zinc finger transcription factor Snail $[9,14]$. At the transcriptional level, Snail is regulated by many signaling pathways including those that are crucial for placental development, such as RAF/MEK/ERK and PI3K/AKT/ mTOR [15]. Post-transcriptional regulation of the highly unstable Snail protein is under control of GSK-3 $\beta$, a member of WNT signaling cascades, and hypoxia-inducible factor alpha (HIF-1 $\alpha$ ) [16,17]. Moreover, Snail expression is also sensitive to hypoxia, either directly through activation of the HIF- $1 \alpha$ responsive element on its promoter, or by such mediators of hypoxic signals as
EGF, TGF $\alpha, \beta$ s and TNF $\alpha$ [14]. Snail up-regulation by HIF-1 $\alpha$ in cultured human first trimester villous explants reduces e-cadherin expression and activates an invasion program [18]. Also, Snail has been shown to down regulate e-cadherin during syncytialization of BeWo trophoblast cell line [19]. In cultured rodent trophoblast cells, Snail expression correlates with the transition from proliferating to terminally differentiated stages [20,21]. It also has been shown that the number of Snail positive EVTs in the basal plate of the human placenta is increased in preeclampsia and HELLP syndrome compared to normal [22], which does not concur with a commonly accepted notion of limited invasive capacity of the EVT in preeclampsia.

Disturbed placenta is believed to be a primary cause of excessive maternal inflammatory response, which in turn instigates endothelial dysfunction and the increased vascular reactivity in preeclampsia [1]. Defects in endothelial function contribute to derangements of sodiumvolume homeostasis and thus compromise cardiovascular adaptations to pregnancy. A variety of animal models which, to a different degree, mirror the vascular pathology seen in human preeclampsia, have been developed [23]. Several studies demonstrated that high sodium uptake during the last week of the rat pregnancy can induce such manifestations of preeclampsia as elevated blood pressure, decreased activity of the renin-angiotensin-aldosterone system, renal dysfunction and fetal growth restriction [24-26]. During the last week of normal rat pregnancy, the trophoblast cells invade the mesometrial triangle, thus stimulating vascular remodeling of maternal arteries through replacement of the maternal endothelium [27]. During the same period, maternal blood pressure gradually falls in spite of blood volume expansion and activation of the renin-angiotensin-aldosterone system [26].

The aim of this study was to examine the protein levels of transcription factor Snail and e-cadherin in placental samples from women with preeclampsia, and from normotensive gestationally-matched preterm and term controls at delivery. We also examined Snail/e-cadherin expression in near-term placentas from rats developing preeclampsia-like symptoms in response to high sodium intake.

\section{Methods \\ Collection of placental tissues}

The human placenta samples were collected at The Toledo Hospital and St. Vincent Mercy Medical Center in Toledo, Ohio, according to approval of the University of Toledo Biomedical Research Review Board, and after informed patient consent. Placental tissues were collected from 9 patients after normal term delivery, from 8 patients with preeclampsia and from 5 patients with 
preterm labor. Pregnancies were diagnosed as preeclamptic according to recommendations given by the American Society of Hypertension [28]. All studied preeclamptic placentas were from women who developed severe hypertension (systolic blood pressure $\geq 160$, diastolic $\geq 110$ ), and heavy proteinuria ( $\geq 4 \mathrm{~g}$ of protein in a 24 hour urine collection, or $\geq 3+$ protein on dipstick measurement on two or more occasions). All 5 preterm deliveries were associated with premature rupture of amniotic membranes. All patients who had normal or premature deliveries did not have any prior diagnosed pregnancy complications. Immediately after delivery, the peripheral parts of placenta, without any visible infarcts or retroplacental clots, were snap frozen in liquid nitrogen or preserved in $10 \%$ formalin.

The salt-induced model of rat preeclampsia was described in detail in several publications [24-26]. Ethical protocol for the rat studies was approved by the Animal Care and Use Committee of the Intramural Research Program, National Institute on Aging [24,29]. Briefly, in the experimental group of Sprague-Dawley pregnant rats, regular drinking water was replaced with water containing $1.8 \% \mathrm{NaCl}$. The salt overdose regime was started at $13 \mathrm{dpc}$ because the last week of normal rat pregnancy is accompanied by blood volume expansion, activation of the renin-angiotensin-aldosterone system and a significant fall in arterial blood pressure. The rat placentas used in the study were extracted from exactly the same control and experimental animal groups (5 and 9 rats respectively) that were thoroughly described earlier [24,29]. After extraction, the whole placentas were snap frozen and sent to our laboratory on dry ice.

\section{Protein extraction}

Large, randomly chosen pieces of human placental tissues (up to $20 \mathrm{~g}$ ) taken from two areas containing both fetal and uterine surfaces, and entire rat placentas were homogenized in liquid nitrogen. The resulting powder was transferred into ice-cold RadioImmunoPrecipitation Assay (RIPA) buffer, containing $50 \mathrm{mM}$ Tris- $\mathrm{HCl}, \mathrm{pH}$ 7.5, $150 \mathrm{mM} \mathrm{NaCl}, 1 \%$ Nonidet P-40, 5\% sodium deoxycholate, $0.1 \%$ SDS, and protease inhibitors (Protease Inhibitor Cocktail, Sigma) and processed as previously described [30].

\section{Western blotting and immunohistochemistry}

Western blotting analysis was carried out using standard protocol as previously described [30]. Briefly, from 10 to $20 \mu \mathrm{g}$ of protein (equal amounts for each gel) were loaded into each well. Proteins were transferred by semi-dry method and then processed exactly as thoroughly described earlier [30]. ImageJ software based analysis (http://rsbweb.nih.gov/ij) was performed to quantify the bands obtained via Western blot analysis.
The area under the curve (AUC) of the specific signal was corrected for the AUC of the actin loading control. The average value for the samples from normal term (human) or control (rat) placentas was set as 1 and other conditions were recalculated correspondingly to allow ratio comparisons.

For double immunohistochemistry of the human placentas, deparaffinized and re-hydrated $5 \mu \mathrm{m}$ thick placental sections were washed in TBS, blocked in TBS containing $10 \%$ goat serum and 5\% BSA for 2 hours at room temperature, and probed with anti-Snail antibodies (ab17732, or ab85935, Abcam, Cambridge, MA, USA) diluted 1:200 in 5\% goat serum/2.5\% BSA in TBS for 1 hour at room temperature and overnight at $4^{\circ} \mathrm{C}$. After washing, the slides were boiled in acetic buffer (Vector Laboratories, Burlingame, CA, USA) in a conventional microwave. Then, the slides were probed with anti-e-cadherin antibodies (BD Bioscience, Sparks, MD, USA) diluted 1:150 in 1.5\% goat serum in TBS. The endogenous peroxidase activity was blocked with $3 \% \mathrm{H}_{2} \mathrm{O}_{2}$ in methanol. E-cadherin antibodies were detected first using anti-mouse HRP-conjugated polymer (Zymed, San-Francisco, CA, USA) for $30 \mathrm{~min}$ followed by the color development with DAB. To detect the anti-Snail antibody, we used anti-rabbit ABC-AP kit and Fast Red chromophore according to standard procedure (Vector Laboratories).

Since whole placentas from experimental rats were utilized for protein extraction, we used commercially available slides of definitive rat placenta for immunohistochemical detection of Snail protein (ab4623, Abcam). The slides were boiled in acetic buffer, and then sections were blocked with $1.5 \%$ goat serum in TBS for 1 hour at room temperature. The tissue sections were first probed with anti-Snail antibody diluted 1:50 in 1.5\% goat serum for 1 hour at room temperature. Afterward, the staining protocol was the same as described for human placenta. Anti-Snail antibodies (ab17732, or ab85935, Abcam) were raised against the same peptide sequence. We tested their specificities by applying the anti-Snail antibodies with this peptide (ab19126, Abcam).

\section{Statistical analysis}

Data are presented as the mean \pm standard error of the mean unless otherwise specified. One-way ANOVA followed by Tukey HSD test was used to compare three groups of human samples. Unpaired Student's t-test was used to evaluate the difference between rat groups. Statistical significance was reported at the ${ }^{*} p<0.05,{ }^{* *} p<$ 0.01 and $* * * \%<0.001$ levels.

\section{Results}

The three groups studied were not significantly different from one another with respect to maternal age at time of delivery (Table 1). Average gestational age was also 
Table 1 Clinical characteristics of patients in the study

\begin{tabular}{lccc}
\hline & Normal term deliveries & Preterm deliveries & Preeclampsia \\
\hline Patients & 9 & 5 & 8 \\
Maternal age (years) & $29.7 \pm 6.4(22-34)$ & $32.6 \pm 7.2(29-38)$ & $25.8 \pm 6.6(20-36)$ \\
Gestational age at & $38.3 \pm 0.5(38-39)$ & $35.4 \pm 1.1(34-36)^{* * *}$ & $31.3 \pm 3.1(28-36)^{* * *}$ \\
deliveries (weeks) & $77.3 \pm 8.2(70-90)$ & $65.0 \pm 26.9(25-90)$ & $39.5 \pm 34.9(5-95)^{*}$ \\
Birth weight (percentile) &
\end{tabular}

Values represent group mean \pm SD. Numbers in the parenthesis show the range of the values

${ }^{*} p<0.05$ preeclampsia versus both preterm and term deliveries

${ }^{* * *} p<0.001$ preterm and preeclampsia deliveries versus normal term

not significantly different between the preterm and preeclampsia groups of patients. Thus, the placenta samples from preterm deliveries served as gestation-matched controls for analysis of Snail/e-cadherin proteins in preeclampsia. The preeclampsia group was heterogeneous with regard to gestational age and fetal birth weight at delivery, yet all samples from preeclamptic placentas were obtained in the preterm period.

Western blotting examination of proteins extracted from human placentas showed that the level of Snail protein was decreased on average by $30 \%$ in preeclampsia compared to normal term (Figure 1). Immunohistochemical analysis demonstrated intensive staining of the Snail protein in the villi of normal term placenta where it was localized in the nuclei of two different cell populations, the mesenchymal cells and VCTs. In the decidua, Snail positive nuclei were found in EVTs which were negative for e-cadherin staining (Figure 2). In line with Western blotting data, the preeclamptic placentas show fewer Snail-positive cells. Western blotting revealed variable expression of Snail among the preterm placentas. No statistically significant differences were found between preterm and term placentas or between preterm and preeclamptic placentas after analysis of Snail expression by Western blotting. However, there was a strong trend toward statistical significance between preterm and preeclamptic placentas (Figure 1).

The expression of e-cadherin was lower (by 30\%) in term placentas than in preterm and preeclamptic placentas as was demonstrated by Western blotting (Figure 1). However, a statistically significant difference in ecadherin expression was found between term and preeclamptic samples only. Reduction in e-cadherin protein level in the term placentas compared to preterm ones is in agreement with widely appreciated phenomenon that the relative number of proliferative VCTs is decreased close to the term [2]. Immunohistochemical analysis demonstrated strong staining for e-cadherin in VCTs in both preterm and preeclamptic placentas (Figure 2). Ecadherin connects neighboring VCT-VCT and VCTSynTB interfaces, and is expressed by proliferative VCTs. E-cadherin is down-regulated during the fusion of more differentiated VCTs to SynTB [31].
Pregnant rats receiving high dose of $\mathrm{NaCl}$ during the last week of gestation develop symptoms of preeclampsia such as high blood pressure, proteinuria, fetal growth restriction, and enhanced vascular reactivity [24-26,29]. Unrelated to human preeclampsia, additional, pathophysiological changes in pregnant rats used in the study included significantly less weight gain ( $7 \%$ vs $\sim 36 \%)$ and fewer (2-3) pups than in normal pregnancy[24,29]. Western blotting analysis demonstrated that in the placentas of rats with salt-induced preeclampsia, Snail protein expression is decreased by $40 \%$ and e-cadherin level increased by $60 \%$ compared to control pregnant rats (Figure 3). Immunohistochemical staining of the rat definitive placenta with anti-Snail antibodies revealed strong nuclear and weak cytoplasmic presence of Snail in spongiotrophoblasts in the junctional zone, and in the sinusoidal trophoblast giant cells (TGC) on the periphery of the labyrinth (Figure 4). Immunohistochemical analysis confirmed that in the rat term placenta e-cadherin is present in the SynTB covering the maternal sinusoides of the labyrinth (Figure 4) [32].

\section{Discussion}

In this study, we have demonstrated that human placentas from patients with preeclampsia contain significantly reduced level of the transcription factor Snail compared with normal term placentas. We also showed that in the human placenta, Snail protein is expressed in the VCTs and mesenchymal cells of the villi, and in the EVTs of the decidua. Our data only partially agree with the previous analysis of Snail protein distribution in the human placenta by Blechschmidt et al. [22]. These authors found strong Snail immunoreactivity in the ETVs of the basal plate and weak Snail immunoreactivity in the VCTs using antibodies which are not recommended specifically for immunohistochemistry ( $\mathrm{Sn} 9 \mathrm{H} 2$, ab31787, Abcam). (We tested this antibody on the human placenta sections and found very weak and irregular immunoreactivity in the deciduas.) Based on calculations of the immunostaining intensity score for e-cadherin and Snail-positive EVTs in the basal plate, Blechschmidt et al. [22] reported an increase in Snail immunoreactivity levels accompanied by a reduction of e-cadherin 


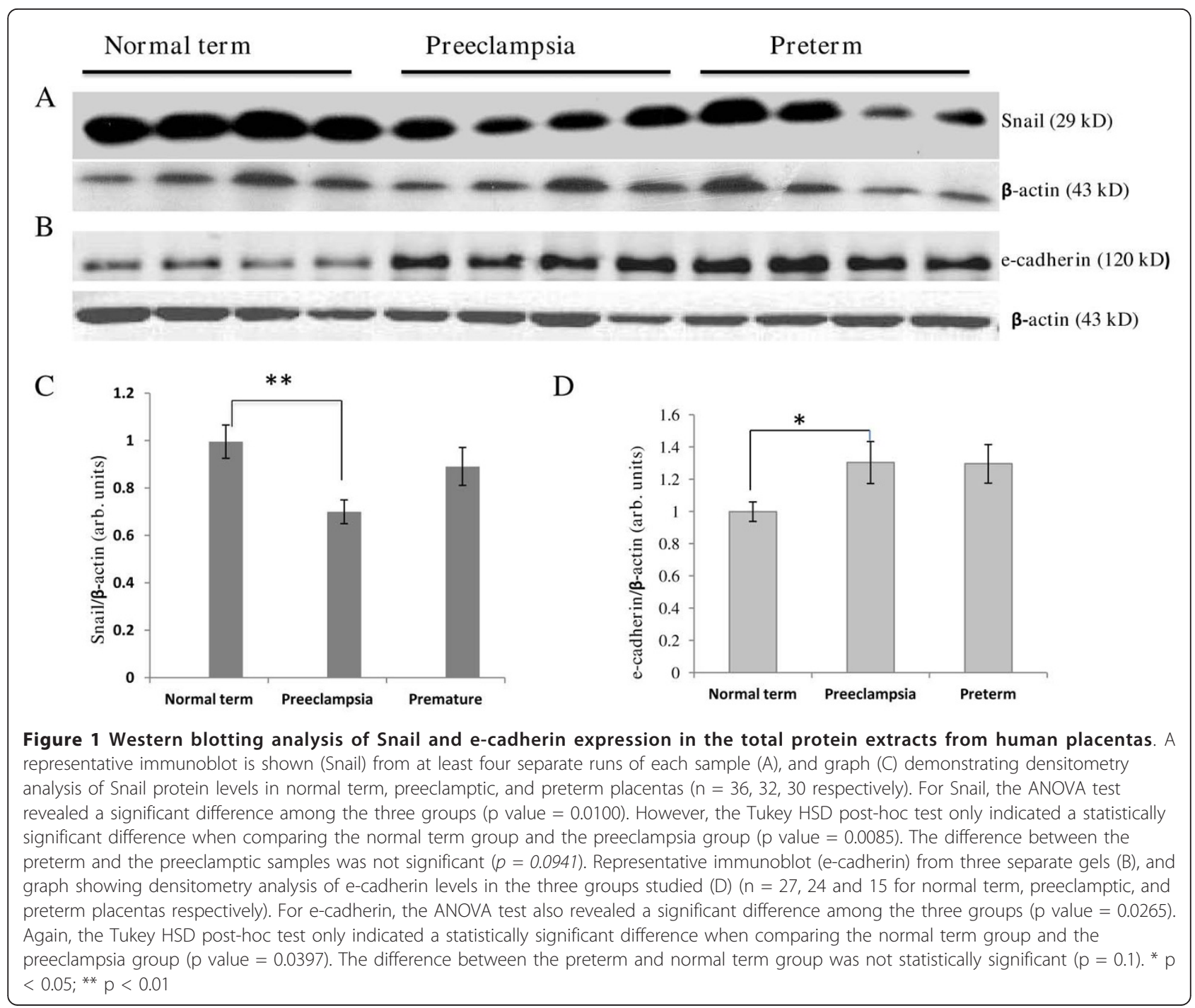

immunoreactivity in preeclamptic placentas compared to normal term placentas. Yet, our observation does not contradict Blechschmidt et al. for at least two reasons. First, we estimated Snail/e-cadherin levels in total homogenates of large pieces of placenta, where the majority of proteins belongs to villous tissue; and second, there is only limited invasion of EVTs in decidual segments of the placental edges [33]. Thus, any changes in Snail/e-cadherin expression in the EVT of the basal plate in our samples of placenta wouldn't significantly alter total levels of these proteins. Our data for e-cadherin expression in the villous compartment of normal and preeclamptic placentas are in agreement with several reports [11,34,35].

Proper spatio-temporal induction, activation and degradation of Snail may be essential for the control of the VCT maturation and thus the maintenance of continuous, uninterrupted syncytium. In addition to e- cadherin repression, Snail regulates cell cycle progression and confers resistance to cell death as has been previously shown for fetal hepatocytes, epithelial cells, and mouse embryos [36-38]. In preeclampsia, the syncytial layer of the villi in some areas is discontinuous, while in others parts it forms the thick aponecrotic knots and sprouts [4]. Mesenchymal cells of the villi, in contrast to the trophoectoderm-derived epithelial-like VCTs, originate from extraembyonic mesoderm, which is formed during primary EMT at the early stages of human development. Thus, villous mesenchymal cells may retain Snail expression from this period onward. The exact role(s) of Snail in the stroma remains to be elucidated and might be related to the regulation of fibroblast/myofibroblast differentiation and angiogenesis. Remarkably, morphologically preeclamptic placentas are distinctive due to the altered branching pattern of the villous tree, particularly when preeclampsia is combined 


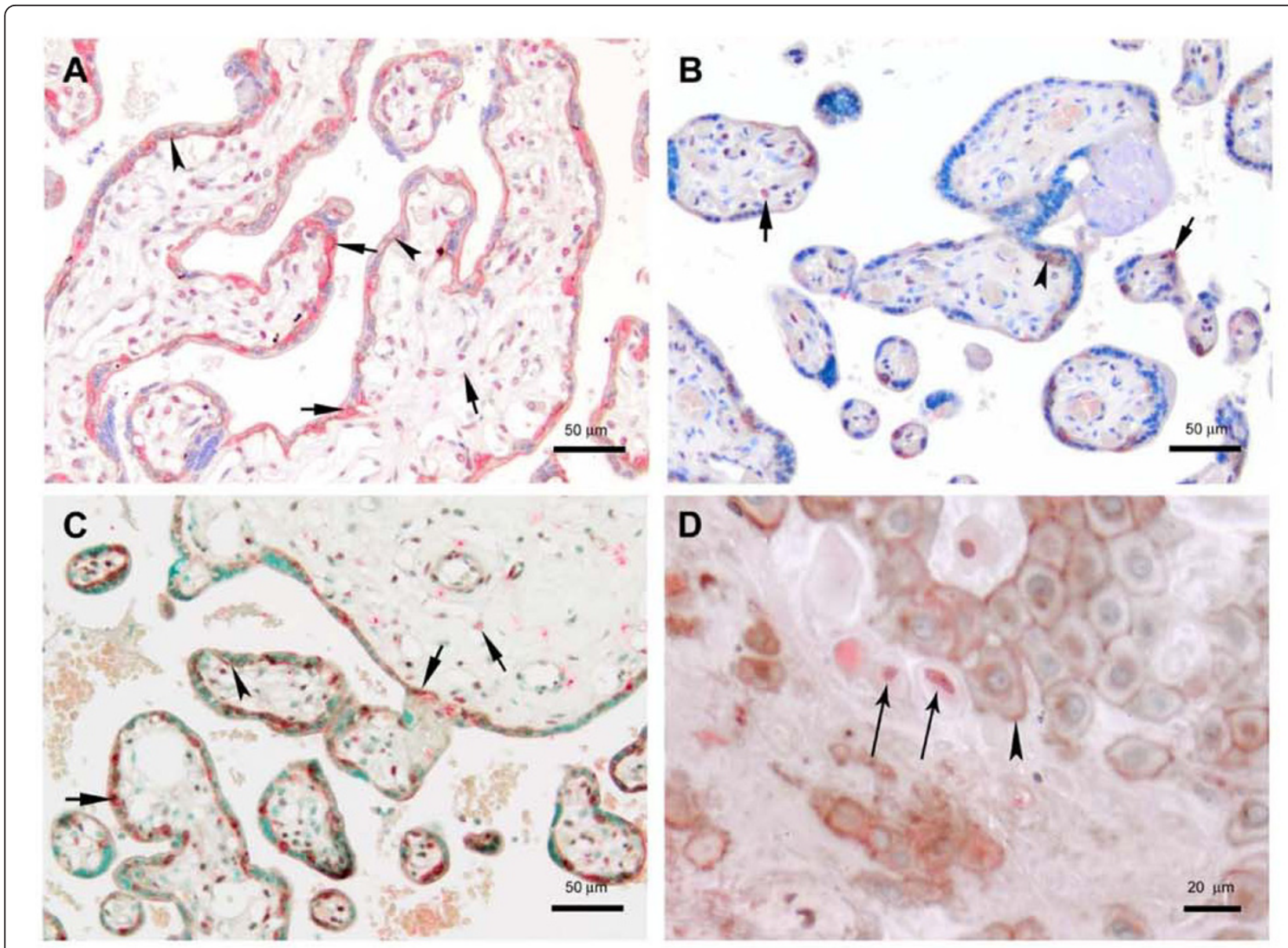

Figure 2 Immunohistochemical analysis of Snail and e-cadherin proteins expression in human normal term, preeclamptic and preterm placentas. E-cadherin (stained brown) is present in the basement membranes of the villi and, to a lesser extent, in the cytoplasm of VCT where it forms a connection between neighboring VCTs, and between VCT and overlying SynTB (arrowheads). Snail (stained red) is localized mostly in the nuclei of VCT and mesenchymal cells (short arrows). A normal placenta (A) and a preeclamptic placenta (B) are shown. In preeclampsia, there is a significant reduction in Snail expression. The premature placenta (C) shows a similar staining pattern to the term placenta. The maternal-fetal interface of a preterm placenta (D) reveals expression of Snail in EVT (long arrows), which lack e-cadherin on their membranes. Note that EVTs which express e-cadherin are Snail negative. The loss of e-cadherin by EVT is believed necessary for proper invasion and implantation

with IUGR $[2,4]$. Thus, down-regulation of Snail by internal and external factors may contribute to the development of human preeclampsia by aberration of essential stages of placental development.

In the rat definitive placenta, Snail is highly expressed in spongiotrophoblasts, the TGC precursor cells in the junctional zone (Figure 4). We also found focal expression of Snail in the sinusoidal TGC at the periphery of the labyrinth. Rodent and human placentas are similar in basic morphology and functions. However, there are some differences in the structure and formation of the chorion [39]. Rodent labyrinth, which is functionally analogous to primate chorionic villi, is lined from the maternal side with one layer of mononuclear sinusoidal TGC and two layers of SynTB. The SynTB contacting fetal mesenchyme originates from chorionic ectoderm enriched with e-cadherin positive progenitor cells. The outer SynTB and sinusoidal TGC are derived from the inner part of the ectoplacental cone where the majority of trophoblast progenitor cells, committed to SynTB fate, persistently express e-cadherin. Before $8 \mathrm{dpc}$ of rat placental development, e-cadherin is localized to trophoblasts; at 9dpc its expression is confined exclusively in trophoblast derived cells at the extraembyonic ectoderm, and from 10dpc onward, e-cadherin remains exclusively expressed in the labyrinth [32]. Thus, in the outer layers of the ectoplacental cone, e-cadherin is lost from $7 \mathrm{dpc}$ onward when most of the Snail-positive cells appear. In situ hybridization analysis demonstrated that from $7.5 \mathrm{dpc}$ Snail transcripts were detected in the ectoplacental cone in precursor cells of TGC and, at much lower levels, in the proliferative trophoblasts of the chorion 
A

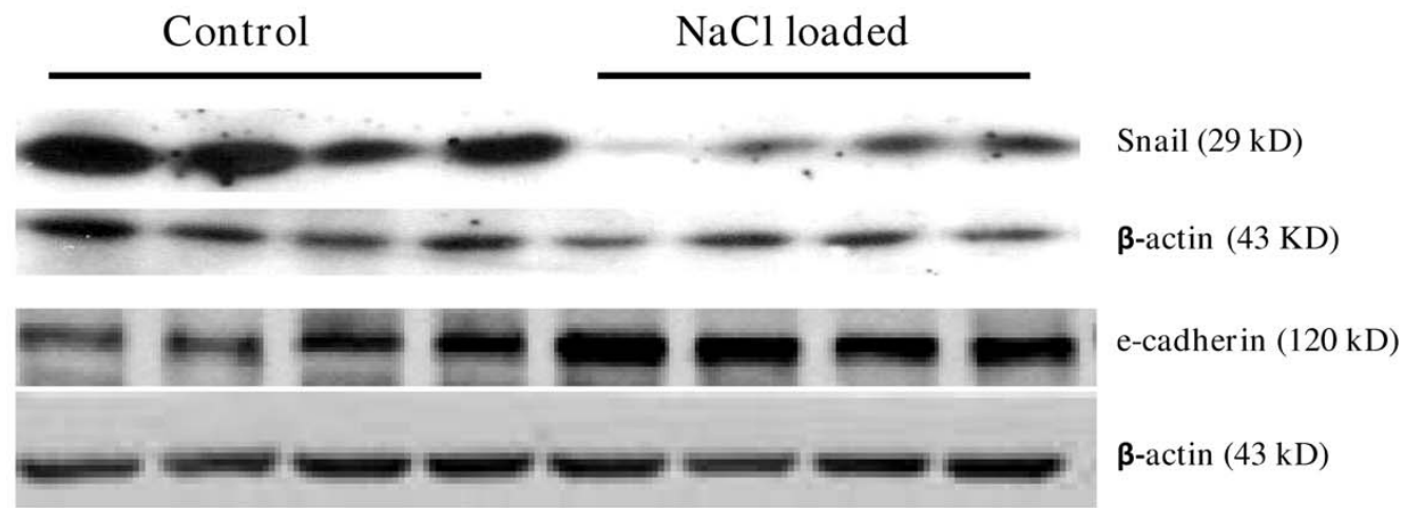

$\mathrm{C}$

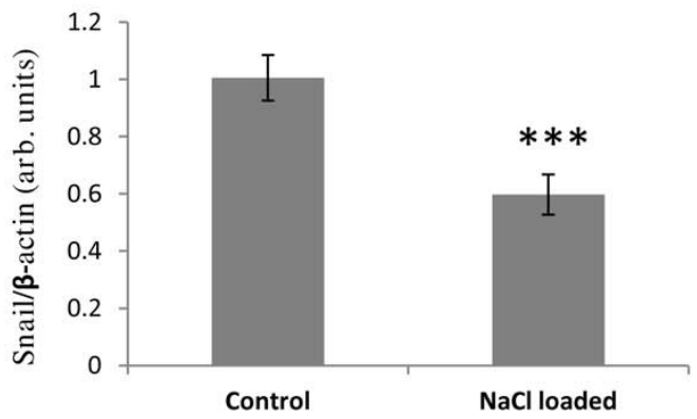

$\mathrm{D}$

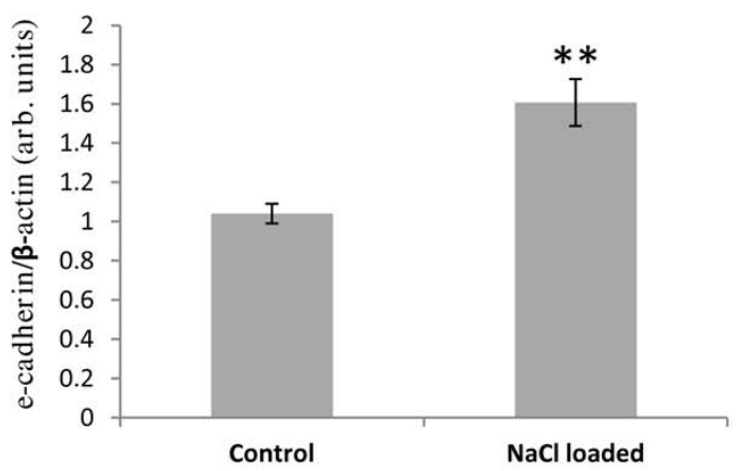

Figure 3 Western blotting analysis of Snail and e-cadherin expression in the total protein extracts from rat placentas. Representative immunoblot from 3 independent runs for each sample (Snail) (A), and graph demonstrating densitometry analysis of Snail protein levels in the placentas of control pregnant rats and rats loaded with $\mathrm{NaCl}(n \geq 15)$ (C). Representative immunoblot from 2 independent runs for each sample (e-cadherin) (B), and graph showing densitometry analysis of e-cadherin levels in the two groups studied $(\mathrm{n} \geq 10)(\mathrm{D}) .{ }^{* *} p<0.01,{ }^{* * *} p<0.001$
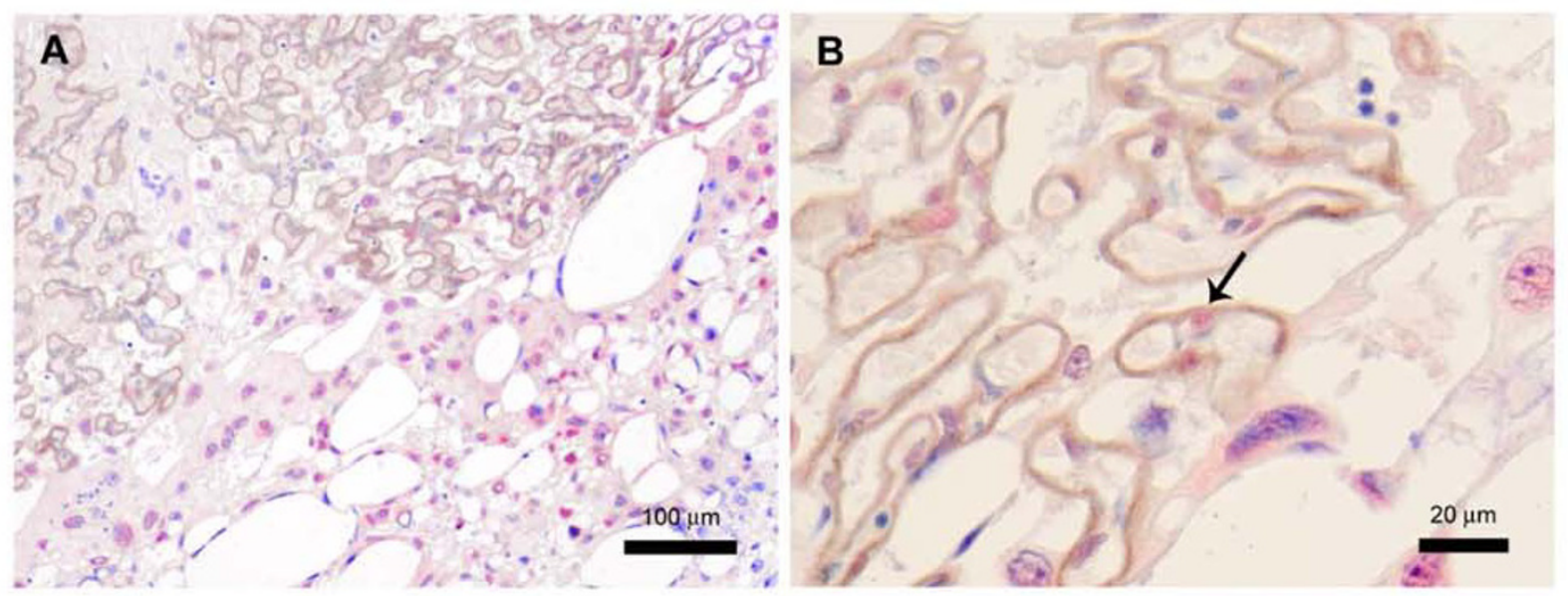

Figure 4 Immunohistochemistry of Snail and e-cadherin expression in normal definitive rat placenta. The photomicrographs show that Snail (stained red) is present in the nuclei of spongiotrophoblast cells of the junctional zone. E-cadherin (stained brown) is localized to the SynTB of the labyrinth (A). Snail positivity is also found in mononuclear sinusoidal TGC in some sinusoids positioned close to the junctional zone (arrow) (B) 
$[40,41]$. At 10.5dpc Snail transcripts were detected in the spongiotrophoblast layer and in some focal areas of the labyrinth [41]. Therefore, only a small number of Snail-positive cells are present in the developing labyrinth before 13dpc. The junctional zone encloses a highly proliferative cell population until day 14 of gestation, afterward the mitotic activity at this zone ceases sharply [42]. Thus, an up-regulation of Snail in the junctional zone spongiotrophoblasts may inhibit their proliferation [36]. Notably, down-regulation of Snail expression takes place at the border between the population of spongiotrophoblasts and endocycling TGC [20]. In Rho-1 trophoblast cell line, down-regulation of Snail promotes entry into endoreduplication through control of the "G2 decision point" [20]. The trophoblasts are the only type of mammalian cells that posses the ability to quit mitosis and enter into endocycle, thus multiplying DNA content in the range $8 \mathrm{~N}-64 \mathrm{~N}$ in the human and up to $4096 \mathrm{~N}$ in rodents [42-44]. These polyploid cells form an invasive front when they contact maternal decidua and play a crucial role in placental development and its communication with maternal tissues [42-44].

In rodents, in contrast to humans, e-cadherin and Snail are persistently expressed in the majority of cells positioned in two different compartments throughout placental development. The only exception is a small number of mononuclear TGC in the sinusoids located close to the junctional zone (Figure 4). Interestingly, SynTB progenitor cells in rodent placenta (like VCT in human) have never been verified [39].

We have also found that Snail is down-regulated and e-cadherin is up-regulated in rat placentas, when pregnant rats developed preeclampsia-like symptoms in response to $\mathrm{NaCl}$ overload. How can the expression of these proteins be coupled if they are expressed by distinct populations of cells? We speculate that decreased levels of Snail in the spongiotrophoblasts of salt-loaded pregnant rats could boost the endoreduplication process, thus amplifying the number of TGC. The TGCs produce activin, a TGF $\beta$ family member protein, which can trigger differentiation of progenitor trophoblast cells away from spongiotrophoblast/TGC fate toward SynTB as suggested in Natalie et al. [45]. Therefore, in the rat, Snail functions appear to be restricted to the control of spongiotrophoblast differentiation.

Snail expression, stability and activity are under control of integrated and complex cellular signaling networks which can be furthermore affected by perturbations in the oxygen level $[14,46]$. Oxidative stress triggers Snail transcription directly through elevation of ROS or by activation of NF- $\kappa$ B signaling pathway $[14,47]$. From the other side, hypoxia promotes Snail protein stability by inhibiting Snail proteasome degradation. HIF- $1 \alpha$ has been shown to down regulate F-box
E3 ubiquitin ligase FBXL14, which targets specifically the cytoplasmic portion of Snail in some cancerous cell lines [17]. Additionally, two highly HIF-up regulated proteins, lysyl oxidase (LOX) and LOX-like 2 (LOXL2) can stabilize Snail and therefore may alter its activity, however, this interaction seems to be cell-line specific $[48,49]$. Interestingly, while hypoxia strongly elevates LOX gene expression, its enzymatic activity requires subsequent re-oxygenation [50]. LOX and LOXL2, which are generally known as classical proteins responsible for the cross linking of collagen and elastin, are normally expressed in the human placenta, and in the VCT and SynTB particularly, throughout gestation [51]. Since preeclamptic placentas are characterized by increased oxidative stress [52], over-expression of active HIF- $1 \alpha$ and 2- $\alpha$ [53] and reduced activity of proteasomal proteins [54], an up-regulation of Snail in human preeclampsia might be suggested. However, the opposite-the reduction of Snail protein-may indicate that other factors associated with pregnancy and/or preeclampsia may have a greater effect on Snail, and thus affect normal placental development. Indeed, compromised oxygenation of placentas in preeclampsia is thought to be secondary to the primary cause of preeclampsia-placental insufficiency due to defective trophoblast functions $[3,6,52,55]$.

The similarity between Snail down-regulation in human preeclampsia and in the rat model may provide additional information about factors which may modulate Snail expression and stability in placentas. The last week of normal rat pregnancy is characterized by expansion of the labyrinth, cessation of spongiotrophoblast proliferation, and TGC invasion of the uterine mesometrial compartment [27]. These developmental events coincide with a significant decrease in maternal blood pressure. Maternal insult in the form of a 20 -fold increase in dietary salt during this period of gestation causes a dramatic elevation of blood pressure accompanied by increased placental oxidative stress and inflammation [25].

Throughout normal pregnancy, maternal blood pressure is decreased despite dramatic increases in extracellular fluid volume and salt retention due to persistent activation of the renin-angiotensin-aldosterone system (RAAS) and placental secretion of the endogenous cardiotonic steroids, inhibitors of Na, K-ATPase [56,57]. RAAS, especially its utero-placental component, has been recently implicated in the pathology of preeclampsia [56]. Particularly, angiotensin II and IV have been shown to regulate invasion, migration and apoptosis of the rat and human trophoblasts [56,58]. These facts suggest that utero-placental and circulating hormones involved in blood pressure control during pregnancy might play a more important role in the direct 
regulation of trophoblast differentiation than has been appreciated.

Although no significant correlations were found between salt consumption and preeclampsia development, some epidemiological studies reported small yet significant correlation between salt intake and hypertension in humans [59]. Recent data demonstrated higher salt sensitivity and pressor response to salt loading in women with a history of hypertensive pregnancy when compared to normotensives [60]. Placental and maternal changes in the salt-induced rat model of preeclampsia mirror many of those noted in humans $[25,26,61,62]$. However, unlike in humans, rats with preeclampsia symptoms due to salt-overload demonstrated large $(\sim 44 \%)$ decrease in food intake, significant reduction of pregnancy-related maternal weight gain and fewer pups $[24,26]$. Caloric restriction of $50 \%$ during the second half of rat pregnancy alone can cause a decrease of maternal and fetal weight and placental alterations [63]. Placenta, and labyrinth specifically, from undernourished pregnant rats have significantly increased apoptosis [64]. However, apoptosis is elevated in a variety of pregnancy complications including preeclampsia [65]. An increased rate of placental apoptosis was shown in the salt-induced rat model as well [25]. Since there are no reports demonstrating caloric restriction as a possible risk factor for preeclampsia development, maternal undernutrition in pregnant rats on a high sodium uptake is one of the limitations of this model and should be considered in the design of future studies.

\section{Conclusions}

This study demonstrates that in the human and rat placentas Snail is expressed in trophoblast and mesenchymal progenitor cell lineages during their transition to the terminally differentiated stage. These observations are congruent with previously reported Snail expression in some of these lineages, such as rat spongiotrophoblasts, human EVT and trophoblast cell lines. We also found that in human preeclampsia and in the rat saltinduced model of preeclampsia, the level of transcription factor Snail is significantly decreased in total placenta extracts. The reduced levels of Snail might trigger alterations in diverse pathways of placental cell lineage differentiation and thus contribute to the development of preeclampsia and/or IUGR.

\section{Acknowledgements}

The authors want to thank Dr. Bagrov and Dr. Fedorova from the Laboratory for Cardiovascular Research, National Institute on Aging for samples of rat placentas. The authors appreciate the help of Dr. Yuing Jiang for assistance with preparation of figures and Dr. Sadik Khuder for statistical analysis. This study was supported by NIH contract \#263-MA-707136-1 (S.J.I. and D.M.) and internal funds at the University of Toledo Medical Center.

\section{Author details}

'Department of Medicine, University of Toledo School of Medicine, Toledo, $\mathrm{OH} 43614$, USA. ${ }^{2}$ Department of Pathology, University of Toledo School of Medicine, Toledo, OH 43614, USA. ${ }^{3}$ Department of Obstetrics and Gynecology, University of Toledo School of Medicine, Toledo, OH 43614, USA.

\section{Authors' contributions}

LF participated in the study design, carried out experiments and prepared the manuscript. CGW performed histopathological and immunohistochemical analysis and contributed to the manuscript preparation. SS and NK collected the human placenta samples, extracted placental proteins and participated in Western blotting analysis. JIS and TH formulated the research questions, conceived the study and participated in drafting of the manuscript. DM participated in the development of critical methodology and the manuscript preparation. All authors read and approved the final manuscript.

\section{Competing interests}

The authors declare that they have no competing interests.

Received: 30 September 2011 Accepted: 23 February 2012

Published: 23 February 2012

\section{References}

1. Steegers EA, von Dadelszen P, Duvekot JJ, Pijnenborg R: Pre-eclampsia. Lancet 2010, 376(9741):631-644

2. Benirschke K, Kaufmann P, Baergen R: Pathology of the human placenta. New York: Springer; 52006.

3. Huppertz B: Placental origins of preeclampsia: challenging the current hypothesis. Hypertension 2008, 51(4):970-975.

4. Burton GJ, Jones CJ: Syncytial knots, sprouts, apoptosis, and trophoblast deportation from the human placenta. Taiwan J Obstet Gynecol 2009, 48(1):28-37.

5. Huppertz B: IFPA Award in Placentology Lecture: biology of the placental syncytiotrophoblast-myths and facts. Placenta 2010, 31(Suppl):S75-S81.

6. Cindrova-Davies T: Gabor Than Award Lecture 2008: pre-eclampsia-from placental oxidative stress to maternal endothelial dysfunction. Placenta 2009, 30(Suppl A):S55-S65.

7. Heazell AE, Lacey HA, Jones CJ, Huppertz B, Baker PN, Crocker IP: Effects of oxygen on cell turnover and expression of regulators of apoptosis in human placental trophoblast. Placenta 2008, 29(2):175-186.

8. Zhou Y, Damsky CH, Fisher SJ: Preeclampsia is associated with failure of human cytotrophoblasts to mimic a vascular adhesion phenotype. One cause of defective endovascular invasion in this syndrome? J Clin Invest 1997, 99(9):2152-2164.

9. Thiery JP, Acloque H, Huang RY, Nieto MA: Epithelial-mesenchymal transitions in development and disease. Cell 2009, 139(5):871-890.

10. Rosario GX, Ain R, Konno T, Soares MJ: Intrauterine fate of invasive trophoblast cells. Placenta 2009, 30(5):457-463.

11. Brown LM, Lacey HA, Baker PN, Crocker IP: E-cadherin in the assessment of aberrant placental cytotrophoblast turnover in pregnancies complicated by pre-eclampsia. Histochem Cell Biol 2005, 124(6):499-506.

12. Zhou Y, Fisher SJ, Janatpour M, Genbacev O, Dejana E, Wheelock M, Damsky CH: Human cytotrophoblasts adopt a vascular phenotype as they differentiate. A strategy for successful endovascular invasion? I Clin Invest 1997, 99(9):2139-2151.

13. Al-Nasiry S, Vercruysse L, Hanssens M, Luyten C, Pijnenborg R: Interstitial trophoblastic cell fusion and E-cadherin immunostaining in the placental bed of normal and hypertensive pregnancies. Placenta 2009, 30(8):719-725

14. Lopez-Novoa JM, Nieto MA: Inflammation and EMT: an alliance towards organ fibrosis and cancer progression. EMBO Mol Med 2009, 1(6-7):303-314.

15. Knofler M: Critical growth factors and signalling pathways controlling human trophoblast invasion. Int J Dev Biol 2010, 54(2-3):269-280.

16. Zhou BP, Deng J, Xia W, XU J, Li YM, Gunduz M, Hung MC: Dual regulation of Snail by GSK-3beta-mediated phosphorylation in control of epithelialmesenchymal transition. Nat Cell Biol 2004, 6(10):931-940.

17. Vinas-Castells R, Beltran M, Valls G, Gomez I, Garcia JM, Montserrat-Sentis B, Baulida J, Bonilla F, de Herreros AG, Diaz VM: The hypoxia-controlled 
FBXL14 ubiquitin ligase targets SNAIL1 for proteasome degradation. J Biol Chem 2010, 285(6):3794-3805.

18. Arimoto-Ishida E, Sakata M, Sawada K, Nakayama M, Nishimoto F, Mabuchi S, Takeda T, Yamamoto T, Isobe A, Okamoto Y, et al: Upregulation of alpha5-integrin by E-cadherin loss in hypoxia and its key role in the migration of extravillous trophoblast cells during early implantation. Endocrinology 2009, 150(9):4306-4315.

19. Butler TM, Elustondo PA, Hannigan GE, MacPhee DJ: Integrin-linked kinase can facilitate syncytialization and hormonal differentiation of the human trophoblast-derived BeWo cell line. Reprod Biol Endocrinol 2009, 7:51.

20. Nakayama H, Scott IC, Cross JC: The transition to endoreduplication in trophoblast giant cells is regulated by the mSNA zinc finger transcription factor. Dev Biol 1998, 199(1):150-163.

21. Gultice AD, Selesniemi KL, Brown TL: Hypoxia inhibits differentiation of lineage-specific Rcho-1 trophoblast giant cells. Biol Reprod 2006, 74(6):1041-1050

22. Blechschmidt K, Mylonas I, Mayr D, Schiessl B, Schulze S, Becker KF, Jeschke U: Expression of E-cadherin and its repressor snail in placental tissue of normal, preeclamptic and HELLP pregnancies. Virchows Arch 2007, 450(2):195-202

23. MCCarthy FP, Kingdom JC, Kenny LC, Walsh SK: Animal models of preeclampsia; uses and limitations. Placenta 2011, 32(6):413-419.

24. Fedorova OV, Kolodkin NI, Agalakova NI, Namikas AR, Bzhelyansky A, StLouis J, Lakatta EG, Bagrov AY: Antibody to marinobufagenin lowers blood pressure in pregnant rats on a high $\mathrm{NaCl}$ intake. J Hypertens 2005, 23(4):835-842.

25. Beausejour A, Bibeau K, Lavoie JC, St-Louis J, Brochu M: Placental oxidative stress in a rat model of preeclampsia. Placenta 2007, 28(1):52-58.

26. Beausejour A, Auger K, St-Louis J, Brochu M: High-sodium intake prevents pregnancy-induced decrease of blood pressure in the rat. Am J Physiol Heart Circ Physiol 2003, 285(1):H375-H383.

27. Vercruysse L, Caluwaerts S, Luyten C, Pijnenborg R: Interstitial trophoblast invasion in the decidua and mesometrial triangle during the last third of pregnancy in the rat. Placenta 2006, 27(1):22-33.

28. Lindheimer MD, Taler SJ, Cunningham FG: Hypertension in pregnancy. J Am Soc Hypertens 2008, 2(6):484-494.

29. Fedorova OV, Simbirtsev AS, Kolodkin NI, Kotov AY, Agalakova NI, Kashkin VA, Tapilskaya NI, Bzhelyansky A, Reznik VA, Frolova EV, et al: Monoclonal antibody to an endogenous bufadienolide, marinobufagenin, reverses preeclampsia-induced Na/K-ATPase inhibition and lowers blood pressure in $\mathrm{NaCl}-$ sensitive hypertension. $J$ Hypertens 2008, 26(12):2414-2425.

30. Fedorova LV, Raju V, El-Okdi N, Shidyak A, Kennedy DJ, Vetteth S, Giovannucci DR, Bagrov AY, Fedorova OV, Shapiro Jl, et al: The cardiotonic steroid hormone marinobufagenin induces renal fibrosis: implication of epithelial-to-mesenchymal transition. Am J Physiol Renal Physiol 2009, 296(4):F922-F934.

31. Aplin JD, Jones CJ, Harris LK: Adhesion molecules in human trophoblast-a review. I. Villous trophoblast. Placenta 2009, 30(4):293-298.

32. Reuss B, Hellmann P, Dahl E, Traub O, Butterweck A, Grummer R, Winterhager E: Connexins and E-cadherin are differentially expressed during trophoblast invasion and placenta differentiation in the rat. Dev Dyn 1996, 205(2):172-182.

33. Vicovac L, Aplin JD: Epithelial-mesenchymal transition during trophoblast differentiation. Acta Anat (Basel) 1996, 156(3):202-216.

34. Benian A, Madazli R, Aksu F, Uzun H, Aydin S: Plasma and placental levels of interleukin-10, transforming growth factor-beta1, and epithelialcadherin in preeclampsia. Obstet Gynecol 2002, 100(2):327-331.

35. Li HW, Cheung AN, Tsao SW, Cheung AL, WS O: Expression of e-cadherin and beta-catenin in trophoblastic tissue in normal and pathological pregnancies. Int J Gynecol Pathol 2003, 22(1):63-70.

36. Vega S, Morales AV, Ocana OH, Valdes F, Fabregat I, Nieto MA: Snail blocks the cell cycle and confers resistance to cell death. Genes Dev 2004, 18(10):1131-1143.

37. Jamora C, Lee P, Kocieniewski P, Azhar M, Hosokawa R, Chai Y, Fuchs E: A signaling pathway involving TGF-beta2 and snail in hair follicle morphogenesis. PLOS Biol 2005, 3(1):e11.

38. Valdes F, Alvarez AM, Locascio A, Vega S, Herrera B, Fernandez M, Benito M, Nieto MA, Fabregat I: The epithelial mesenchymal transition confers resistance to the apoptotic effects of transforming growth factor Beta in fetal rat hepatocytes. Mol Cancer Res 2002, 1(1):68-78.
39. Georgiades P, Ferguson-Smith AC, Burton GJ: Comparative developmental anatomy of the murine and human definitive placentae. Placenta 2002, 23(1):3-19.

40. Nieto MA, Bennett MF, Sargent MG, Wilkinson DG: Cloning and developmental expression of Sna, a murine homologue of the Drosophila snail gene. Development 1992, 116(1):227-237.

41. Smith DE, Del Amo Franco F, Gridley T: Isolation of Sna, a mouse gene homologous to the Drosophila genes snail and escargot: its expression pattern suggests multiple roles during postimplantation development. Development 1992, 116(4):1033-1039.

42. Zybina TG, Zybina EV: Cell reproduction and genome multiplication in the proliferative and invasive trophoblast cell populations of mammalian placenta. Cell Biol Int 2005, 29(12):1071-1083.

43. Zybina TG, Kaufmann P, Frank HG, Freed J, Kadyrov M, Biesterfeld S: Genome multiplication of extravillous trophoblast cells in human placenta in the course of differentiation and invasion into endometrium and myometrium. I. Dynamics of polyploidization. Tsitologiia 2002, 44(11):1058-1067.

44. Zybina TG, Frank HG, Biesterfeld S, Kaufmann P: Genome multiplication of extravillous trophoblast cells in human placenta in the course of differentiation and invasion into endometrium and myometrium. II. Mechanisms of polyploidization. Tsitologiia 2004, 46(7):640-648.

45. Natale DR, Hemberger M, Hughes M, Cross JC: Activin promotes differentiation of cultured mouse trophoblast stem cells towards a labyrinth cell fate. Dev Biol 2009, 335(1):120-131.

46. Nieto MA: The ins and outs of the epithelial to mesenchymal transition in health and disease. Annu Rev Cell Dev Biol 2011, 27:347-376.

47. Radisky DC, Levy DD, Littlepage LE, Liu H, Nelson CM, Fata JE, Leake D, Godden EL, Albertson DG, Nieto MA, et al: Rac1b and reactive oxygen species mediate MMP-3-induced EMT and genomic instability. Nature 2005, 436(7047):123-127.

48. Peinado H, Del Carmen Iglesias-de la Cruz M, Olmeda D, Csiszar K, Fong KS, Vega S, Nieto MA, Cano A, Portillo F: A molecular role for lysyl oxidaselike 2 enzyme in snail regulation and tumor progression. EMBO J 2005, 24(19):3446-3458.

49. Schietke R, Warnecke C, Wacker I, Schodel J, Mole DR, Campean V, Amann K, Goppelt-Struebe M, Behrens J, Eckardt KU, et al: The lysyl oxidases LOX and LOXL2 are necessary and sufficient to repress Ecadherin in hypoxia: insights into cellular transformation processes mediated by HIF-1. J Biol Chem 2010, 285(9):6658-6669.

50. Postovit LM, Abbott DE, Payne SL, Wheaton WW, Margaryan NV, Sullivan R, Jansen MK, Csiszar K, Hendrix MJ, Kirschmann DA: Hypoxia/reoxygenation: a dynamic regulator of lysyl oxidase-facilitated breast cancer migration. J Cell Biochem 2008, 103(5):1369-1378.

51. Hein S, Yamamoto SY, Okazaki K, Jourdan-LeSaux C, Csiszar K, BryantGreenwood GD: Lysyl oxidases: expression in the fetal membranes and placenta. Placenta 2001, 22(1):49-57.

52. Burton GJ, Hung TH, Jauniaux E: Placental hypoxia, hyperoxia and ischemia-reperfusion injury in preeclampsia. In Preeclampsia Etiology and Clinical Practice. Edited by: Belfort LF. Cambridge: Cambridge University Press; 2007:138-151.

53. Rajakumar A, Brandon HM, Daftary A, Ness R, Conrad KP: Evidence for the functional activity of hypoxia-inducible transcription factors overexpressed in preeclamptic placentae. Placenta 2004, 25(10):763-769.

54. Rajakumar A, Michael HM, Daftary A, Jeyabalan A, Gilmour C, Conrad KP: Proteasomal activity in placentas from women with preeclampsia and intrauterine growth restriction: implications for expression of HIF-alpha proteins. Placenta 2008, 29(3):290-299.

55. Huppertz B: Placental pathology in pregnancy complications. Thromb Res 2011, 127(Suppl 3):S96-S99.

56. Herse F, Dechend R, Harsem NK, Wallukat G, Janke J, Qadri F, Hering L, Muller DN, Luft FC, Staff AC: Dysregulation of the circulating and tissuebased renin-angiotensin system in preeclampsia. Hypertension 2007, 49(3):604-611

57. Fedorova OV, Tapilskaya NI, Bzhelyansky AM, Frolova EV, Nikitina ER, Reznik VA, Kashkin VA, Bagrov AY: Interaction of Digibind with endogenous cardiotonic steroids from preeclamptic placentae. J Hypertens 2009, 28(2):361-366.

58. Williams PJ, Mistry HD, Innes BA, Bulmer JN, Pipkin FB: Expression of AT1R, AT2R and AT4R and their roles in extravillous trophoblast invasion in the human. Placenta 2010, 31(5):448-455. 
59. Karppanen H, Mervaala E: Sodium intake and hypertension. Prog Cardiovasc Dis 2006, 49(2):59-75.

60. Saxena AR, Karumanchi SA, Brown NJ, Royle CM, McElrath TF, Seely EW: Increased sensitivity to angiotensin II is present postpartum in women with a history of hypertensive pregnancy. Hypertension 2010, 55(5):1239-1245.

61. St-Louis J, Sicotte B, Beausejour A, Brochu M: Remodeling and angiotensin II responses of the uterine arcuate arteries of pregnant rats are altered by low-and high-sodium intake. Reproduction 2006, 131(2):331-339.

62. Beausejour A, Houde V, Bibeau K, Gaudet R, St-Louis J, Brochu M: Renal and cardiac oxidative/nitrosative stress in salt-loaded pregnant rat. Am J Physiol Regul Integr Comp Physiol 2007, 293(4):R1657-R1665.

63. Belkacemi L, Nelson DM, Desai M, Ross MG: Maternal undernutrition influences placental-fetal development. Biol Reprod 2010, 83(3):325-331.

64. Belkacemi L, Desai M, Michael Nelson D, Ross MG: Altered mitochondrial apoptotic pathway in placentas from undernourished rat gestations. Am J Physiol Regul Integr Comp Physiol 2011, 301(6):R1599-1615.

65. Sharp AN, Heazell AE, Crocker IP, Mor G: Placental apoptosis in health and disease. Am J Reprod Immunol 2010, 64(3):159-169.

doi:10.1186/1477-7827-10-15

Cite this article as: Fedorova et al: Down-regulation of the transcription factor snail in the placentas of patients with preeclampsia and in a rat model of preeclampsia. Reproductive Biology and Endocrinology 2012 10:15.

\section{Submit your next manuscript to BioMed Central and take full advantage of:}

- Convenient online submission

- Thorough peer review

- No space constraints or color figure charges

- Immediate publication on acceptance

- Inclusion in PubMed, CAS, Scopus and Google Scholar

- Research which is freely available for redistribution

Submit your manuscript at www.biomedcentral.com/submit 